\title{
Diacronie
}

Studi di Storia Contemporanea

$\mathrm{N}^{\circ} 36,4 \mid 2018$

Viaggi e turismo nell'Europa del Novecento

\section{Le tourisme des Français en RDA: à la rencontre de la Prusse Rouge}

William Richier

\section{Q OpenEdition}

1 Journals

\section{Édition électronique}

URL : https://journals.openedition.org/diacronie/10251

DOI : 10.4000/diacronie. 10251

ISSN : 2038-0925

Éditeur

Association culturelle Diacronie

Référence électronique

William Richier, «Le tourisme des Français en RDA: à la rencontre de la Prusse Rouge », Diacronie [En ligne], $N^{\circ} 36,4$ | 2018, document 8, mis en ligne le 29 décembre 2018, consulté le 28 juin 2022. URL : http://journals.openedition.org/diacronie/10251 ; DOI : https://doi.org/10.4000/diacronie.10251 


\section{Diacronie}

Studi di Storia Contemporanea

$36,4 / 2018$

Viaggi e turismo nell'Europa del Novecento

\section{Le tourisme des Français en RDA: à la rencontre de la Prusse Rouge}

\section{William RICHIER}

Per citare questo articolo:

RICHIER, William, «Le tourisme des Français en RDA: à la rencontre de la Prusse Rouge», Diacronie. Studi di Storia Contemporanea : Viaggi e turismo nell'Europa del Novecento, 36, 4/2018, 29/12/2018,

URL: < http://www.studistorici.com/2018/12/29/richier_numero_36/ >

Diacronie Studi di Storia Contemporanea $\rightarrow$ http://www.diacronie.it

Rivista storica online. Uscita trimestrale.

redazione.diacronie@hotmail.it

Comitato di direzione: Naor Ben-Yehoyada - João Fábio Bertonha - Christopher Denis-Delacour - Maximiliano Fuentes Codera Anders Granås Kjøstvedt - John Paul Newman - Deborah Paci - Niccolò Pianciola - Spyridon Ploumidis - Wilko Graf Von Hardenberg

Comitato di redazione: Jacopo Bassi - Luca Bufarale - Gianluca Canè - Luca G. Manenti - Fausto Pietrancosta - Alessandro Salvador - Matteo Tomasoni - Luca Zuccolo

Diritti: gli articoli di Diacronie. Studi di Storia Contemporanea sono pubblicati sotto licenza Creative Commons 3.0. Possono essere riprodotti e modificati a patto di indicare eventuali modifiche dei contenuti, di riconoscere la paternità dell'opera e di condividerla allo stesso modo. La citazione di estratti è comunque sempre autorizzata, nei limiti previsti dalla legge. 


\title{
8/ Le tourisme des Français en RDA: à la rencontre de la Prusse Rouge
}

\author{
William RICHIER
}

L'année 1959 est celle des premiers jumelages entre des municipalités communistes françaises et des villes estallemandes sous l'égide de la Fédération mondiale des villes jumelées. Les Echanges franco-allemands (EFA) sont créés en 1958. Les jumelages avec la RDA et les EFA sont contrôlés par le Parti communiste français. Les témoignages des visiteurs français qui se rendent en RDA reflètent des impressions très différentes, de la séduction totale au rejet. L'Allemagne de l'Est tente d'exporter jusqu'à sa disparition en 1990 une image de bismarckisme socialiste ou de Prusse Rouge: un Etat socialiste et bourgeois, démocratique et autoritaire, pacifiste et militariste contre le prétendu fascisme ouest-allemand.

Les jumelages entre municipalités d'Europe se sont essentiellement développés après la Seconde guerre mondiale. Antoine Vion ${ }^{1}$ rappelle qu'ils doivent beaucoup aux fédéralistes européens qui créent en décembre 1946 à Paris l'Union européenne des fédéralistes. Alexandre Marc, Denis de Rougemont, Altiero Spinelli, André Voisin et Henri Frenay ont joué un rôle particulièrement actif au sein de cette organisation. Plusieurs associations de jumelage se constituent comme le Conseil des communes d'Europe né à Genève en 1951 à l'initiative des fédéralistes européens. Il encourage notamment les appariements entre communes françaises et ouest-allemandes. Le Traité de l'Elysée donnera une impulsion décisive aux jumelages entre la France et la RFA. Ainsi, en 1971, les jumelages franco-allemands représentaient $69 \%$ des jumelages européens ${ }^{2}$.

Le Monde bilingue fondé en 1951 à Paris par Jean-Marie Bressand, Henry Frenay, André Voisin etc, cherche à promouvoir le bilinguisme par les jumelages entre la France, la Grande-Bretagne et les Etats-Unis.

\footnotetext{
${ }^{1}$ VION, Antoine, «L'invention de la tradition des jumelages (1951-1956): mobilisations pour un droit», in Revue française de science politique, 5/2003, pp. 559-582.

2 DEFRANCE, Corine, «Les jumelages franco-allemands. Aspect d'une coopération transnationale», in Vingtième Siècle. Revue d'histoire, 99, 3/2008, pp. 189-201, p. 190.
} 
En 1956 le Monde bilingue, qui subit l'influence croissante du PCF, devient la Fédération mondiale des villes jumelées afin de développer les jumelages en direction du Bloc de l'Est et du Tiers-Monde. Ainsi l'URSS, qui prône la Coexistence pacifique, intègre progressivement les jumelages à sa politique extérieure.

En 1958, une association se créé sous le nom d'Échanges Franco-Allemands (EFA) pour intensifier les relations d'amitié avec la République Démocratique Allemande. Elle se fixe pour objectif de développer les contacts culturels, économiques, les jumelages avec l'Allemagne socialiste. Elle espère ainsi pousser la France à reconnaître un Etat que les Occidentaux considèrent comme la zone d'occupation soviétique et qu'ils appellent « République de Pankow ».

Les EFA sont nés de la coexistence pacifique. Ils se prétendent apolitiques et refusent de porter un jugement sur le régime est-allemand. L'association cherche à attirer les hommes d'affaires, la gauche non-communiste et les chrétiens sans les contraindre à prendre officiellement parti entre les deux blocs. En pratique les EFA sont un satellite du PCF et agissent de concert avec les militants communistes de la Fédération mondiale des villes jumelées, organisation française qui, encourage les jumelages avec des villes de l'Est. Les EFA et les comités de jumelage promeuvent une forme de tourisme politique des Français en RDA que nous allons étudier. Nous empruntons la notion de Prusse Rouge au livre de Nicolas Martin ${ }^{3}$. Nous y ajoutons comme synonyme, celle de socialisme bismarckien. Tandis que la RFA connaissait progressivement la société de consommation et s'américanisait, la RDA apparaissait comme un conservatoire des valeurs d'autorités du II ème Reich.

Entre 1959 et l'année 1973 les voyages de Français en RDA se multiplient dans un pays mal connu. En 1973 la France reconnaît la RDA et le tourisme peut sembler plus facile. A partir de 1981 la France accentue son rapprochement vis-à-vis de la RDA.

\section{Tourisme et voyage dans un pays mal connu (1959-1973)}

\subsection{Les structures du tourisme en RDA}

Les EFA et les comités de jumelage organisent les voyages de Français en RDA en se calquant sur France-URSS et l'organisation soviétique. Le parallèle entre la revue « Les Rencontres francoallemandes » et le livre de Sophie Coeuré et Rachel Mazuy Cousu de fil rouge ${ }^{4}$ atteste que le régime est-allemand n'a rien inventé.

\footnotetext{
${ }^{3}$ MARTIN, Nicolas, La Prusse Rouge, 24 ans de socialisme à la prussienne, Paris, Presses de la cité, 1973.

${ }^{4}$ CEEURÉ, Sophie, MAZUY, Rachel (dir.), Cousu de fil rouge: voyages des intellectuels français en Union Soviétique; 150 documents des archives russes, Paris, CNRS Éditions, 2012.
} 
Le comité national des EFA a recruté au sein de l'intelligentsia non communiste à l'image du Cercle de la Russie neuve créée en 1928. Dans son acception soviétique l'intelligentsia est une notion floue qui regroupe les intellectuels, les artistes, les techniciens et les cadres.

Leurs noms sont mélangés à ceux des communistes. On trouve ainsi des mendésistes comme André Hauriou, Charles Hernu, Gilbert Martin, ou encore le général Billotte gaulliste de gauche. Le Comité national compte des artistes non communistes, comme Jean Cocteau, ainsi que des compagnons de route, sympathisants et membres du Parti à l'exemple d'Ernest Labrousse, de l'écrivain Adamov, du cinéaste Louis Daquin, du compositeur Louis Durey ou de Pierre Abraham directeur de la revue Europe. Le PCF dirige l'Agence littéraire et artistique parisienne (ALAP) cofondée par Louis Aragon, Jean Lurçat et Georges Soria. Les EFA reprennent ainsi la diplomatie culturelle qu'avait intensément engagée Moscou de 1930 à 1937. Les entrepreneurs, les techniciens et les milieux d'affaires étaient activement courtisés par l'URSS avant la guerre. Ils sont discrètement représentés au sein des EFA. Le sénateur Gauche démocratique Etienne Restat est vice-président de la Commission des Affaires économiques et du Plan. Gilbert Martin est président de la Chambre d'Agriculture de l'Eure et président du Comité de vulgarisation du progrès agricole. La loi d'orientation agricole est programmée. Elle sera votée en 1960. Les voyages prévus par les EFA et les jumelages visent des échanges technologiques et des fins de renseignement. L'agriculture, comme l'industrie et les services constituent l'un des secteurs des économies de l'Est où les retards sont les plus graves.

\subsection{Le tourisme économique, politique et social}

\subsubsection{Un second miracle économique allemand?}

La RDA cherche à attirer des entrepreneurs et des élus chargés des questions économiques. Le polytechnicien Henri Jannès, chargé de mission auprès de la commission des finances du Sénat a visité « l'exposition de la standardisation » qui s'est tenue à Leipzig en 1959. Il en a tiré un rapport intitulé « l'Allemagne de l'est est-elle au seuil d'une révolution industrielle ?». Depuis le début du Premier plan quinquennal la production industrielle a plus que doublé et le développement économique de la RDA a été plus rapide que celui de la RFA. Il affirme que la RDA joue un rôle de plus en plus important dans le commerce mondial. Henri Jannes omet qu'elle est tournée vers le bloc de l'Est dont il surestime les performances économiques. Elle bénéficie de surcroît de ses échanges avec la RFA. Il note : 
«Ou bien les statistiques de la RDA sont fausses et il est difficile de penser qu'un Etat risque sa réputation à ce jeu ou bien la RDA est en pleine ascension économique » ${ }^{5}$. Et encore : « N’y auraitil non pas un mais deux miracles allemands dont celui dont on ne parle pas serait peut-être encore plus extraordinaire que l'autre $»^{6}$. Il décrit une économie planifiée qui assure le pleinemploi. Dans son numéro de novembre 1960 la revue publie un extrait de son rapport. Il note :

C'est donc une doctrine technico-économique qui est ainsi enseignée : cette doctrine [...] tient en trois points : standardisation - spécialisation - automatisation.

Il s'agit bien d'une doctrine, et non pas d'un programme, ni d'un plan ; nulle part il n'est dit : en telle année, nous produirons tant de tonnes de tel ou tel produit ; cette doctrine au surplus est technique et non pas politique. Nulle part il n'est question de Karl Marx et, effectivement, on ne trouve pas dans l'œuvre ce celui-ci aucun des trois maitres-mots de la nouvelle doctrine: "standardisation, spécialisation, automatisation". Au surplus il s'agit d'idées qui n'étaient pas encore nées au XIXème siècle ${ }^{7}$.

Le Bloc de l'Est cherche à attirer des investisseurs occidentaux. La RDA qui est l'Etat le plus jeune du camp socialiste leur sert de vitrine. Afin de séduire les experts économiques comme le polytechnicien Henri Jannès elle adopte leur discours sur la fin des idéologies. Elle gomme ainsi le caractère communiste de son économie et donne l'illusion d'être une RFA socialisante. Nous parlerons de socialisme bismarckien ou de bismarckisme socialiste pour nommer cette image que cherche à exporter la RDA auprès des Français. Le Bloc de l'Est a pris la mesure des témoignages désastreux comme Retour de l'URSS d'André Gide ${ }^{8}$.

En 1961 Georges Castellan confirme qu'il a vu au cours de l'été précédent des files d'attente à Leipzig devant les boutiques de fruits et légumes. Il note :

Ce n'est un secret pour personne que la RDA comme l'Allemagne Fédérale ne se suffit pas en ce domaine. Un à-coup dans les importations peut donc engendrer une pénurie temporaire. Et ceux qui ont franchi le Rhin une fois savent aussi la manie allemande des "vitamines". Toute raréfaction est sentie comme une frustration. Les queues de Leipzig, pour regrettable qu'elles aient été, et les autorités s'en préoccupèrent, ne mettaient pas en danger la santé des Saxons. Mais une certaine propagande en tira une vision de famine ${ }^{9}$.

\footnotetext{
${ }^{5}$ « Rapport du secrétaire général », in Rencontres franco-allemandes, 5, 1960, p. 3.

${ }^{6}$ Ibidem.

${ }^{7}$ JANNÈS, Henri, « Vers l'automation complète en RDA», in Rencontres franco-allemandes, 7, 1960.

${ }^{8}$ GIDE, André, Retour de l'URSS, Paris, Gallimard, 1936.

${ }^{9}$ CASTELLAN, Georges, «L'Eglise en RDA», in Rencontres franco-allemandes, 10, 1961, p. 3.
} 
En 1958 Walter Ulbricht a engagé la marche forcée vers le socialisme. La collectivisation a désorganisé la production agricole. L'historien Georges Castellan est membre de la présidence collective des EFA. Il est convaincu des performances économiques de la RDA.

Le numéro 15 des «Rencontres franco-allemandes» d'octobre 1962 évoque le voyage d'études qu'on effectué douze députés français en RDA en septembre.

Jean Delrez député MRP se définit comme libre-échangiste. Il note :

Ce que j'ai vu ne m'a pas amené cependant à considérer que le remplacement du système économique capitaliste par un système socialiste puisse être considéré comme un facteur de rétrogradation économique, au contraire, la planification systématique et autoritaire, l'orientation possible de l'économie vers des facteurs individuels essentiels et celle de besoins collectifs trop souvent négligés sont incontestablement des facteurs de croissance économique, des mécanismes "anti-crise" efficaces, des possibilités de satisfaire des besoins sociaux trop souvent négligés au profit des "gadgets" dénoncés notamment par l'économiste américain Galbraith.

Ce sont ces réflexions qui m'ont donné l'explication d'une certaine pénurie que j'ai décelée au cours de mon voyage, manque de choix dans les articles exposés dans les magasins, une qualité qui m'a semblé souvent inférieure, des prix qui m'ont paru élevés ${ }^{10}$.

Ces remarques confirment que le Bloc de l'Est a pris la mesure des effets désastreux des retours de voyage comme Retour de l'URSS. Ce raisonnement est symptomatique de la pensée économique des milieux gaullistes, mendésistes, socialistes ou MRP du début des années 1960. La crise des années 1930 a décrédibilisé le néo-libéralisme aux yeux des dirigeants occidentaux. Galbraith, conseiller de Roosevelt, Kennedy et Johnson, exerce une forte influence sur les économistes français, particulièrement au Plan. Ces derniers en quête d'équilibre entre inflation, investissement et croissance, estiment que le service public peut contribuer à contrôler les prix en réduisant la consommation individuelle. Il impute la pénurie à un choix économique et non à la conséquence d'un système économique de type soviétique que la RDA s'efforce de gommer. L'image d'un socialisme bismarckien que s'efforce de promouvoir le régime est-allemand a de quoi séduire les experts du début des années 1960 à la recherche du «carré magique » défini par Kaldor.

Jean Delrez qui a visité le complexe industriel en construction de Hoyerswerda s'étonne que la RDA puisse investir massivement dans une matière aussi pauvre que le lignite dans une période de surabondance de pétrole et de charbon. Il note une insuffisante synchronisation entre les besoins et les possibilités des Etats-membres du COMECON qu'il définit comme le Marché

\footnotetext{
${ }^{10}$ Temoignage de Jean Delrez, in Rencontres franco-allemandes, 15, 1962, p. 5.
} 
Commun des pays de l'Est. S'il paraît séduit par le décollage de la RDA il reste prudent. Il n'est guère surprenant qu'un député MRP de Moselle dont l'économie repose en partie sur la sidérurgie puisse s'intéresser à l'industrialisation d'un pays sorti ruiné de la guerre et prétend se réconcilier avec l'ennemi d'hier, l'URSS pour les Allemands de l'Est.

Henri Collomb, député Indépendant et paysan du Rhône, salue également les efforts accomplis dans les complexes industriels d'Hoyerswerda et de Schwartz-Pump et s'étonne qu'ils visent à exploiter du lignite.

Emile Hémain, député Indépendant et paysan de la Loire, estime que les économies de l'Est ont été dépassées par l'Ouest parce qu'elles ont nationalisé l'intégralité de l'économie, y compris l'artisanat, aux dépens de l'initiative individuelle. Il justifie la nationalisation du gaz, de l'électricité et des chemins de fer. Au début des années 1960 le néo-libéralisme n'a pas bonne presse, même chez les Indépendants et paysans.

Il ressort des rares témoignages de députés que la RDA a obtenu ce qu'elle souhaitait. Elle les a convaincus que sa reconnaissance était une nécessité diplomatique et économique. Le bismarckisme socialiste exerce une certaine séduction.

\title{
1.2.2. Un régime démocratique?
}

Jean Delrez n'est pas dupe de la fiction démocratique du régime. Il note:

\begin{abstract}
les élections, la liste unique présentée par le "Front National", m’ont évidemment laissé assez sceptique sur le fonctionnement d'une démocratie au sein de laquelle l'opposition ne trouve aucune place. Le sens véritable de la différenciation des partis m'a quelque peu échappé, sur 466 députés, la Chambre du Peuple ne comporte que 117 SED (Parti Socialiste Unifié), le reste se répartissant entre 9 autres partis, dont la CDU (Démocratie chrétienne). Les quelques contacts que j'ai eus avec la population m'ont révélé une absence d'adhésion pour le moins importante au régime ${ }^{11}$.
\end{abstract}

\subsubsection{Les relations sociales}

Nestor Rombeaut député MRP de Loire-Atlantique et militant note qu'à Stalinstadt ${ }^{12}$ le syndicat a demandé le licenciement de 60 ouvriers afin d'améliorer la productivité :

\footnotetext{
${ }^{11}$ Ibidem.

${ }^{12}$ Devenue Eisenhüttenstadt en 1961.
} 
L'entreprise est socialiste, me répond-on. C'est vrai ...mais à mes yeux un licenciement demeure toujours une blessure infligée au travailleur et la productivité ne peut pas tout justifier.

Il m'est aussi difficile d'accepter le fait que le travailleur qui est déficient ou défaillant soit signalé à l'attention de ses camarades et de la population par voie d'affiche dans l'usine et éventuellement de la population par la presse ${ }^{13}$.

L'humiliation publique est banale dans les régimes de type soviétique. Elle fait partie du stakhanovisme. Dès sa naissance la RDA a lancé le mouvement d'émulation socialiste en citant en exemple Adolf Hennecke. Le 13 octobre est le jour anniversaire des activistes en Allemagne de l'Est. Le dépassement de normes inatteignables permet d'exploiter et de terroriser les ouvriers. En juin 1953 l'augmentation des cadences avait suscité le soulèvement de Berlin-Est. En 1961 le régime renforce la discipline dans les usines et impose des obligations de rendement. Le témoignage de Nestor Rombeaut n'a rien de surprenant. L'Etat ouvrier craint paradoxalement les ouvriers.

\subsubsection{L'Etat anti-fasciste allemand}

C'est peut-être le discours anti-fasciste qui convainc le moins les visiteurs français.. Jean Delrez s'avoue « désagréablement surpris » que la propagande présente Adenauer comme un nazi.

En juin 1963 Jean Le Lann député centre démocratique de Fougères évoque un jeune officier qui:

nous a fait un vrai discours de guerre (et avec quelle conviction!) à la porte de Brandebourg. Si beaucoup de jeunes sont ainsi éduqués on risque d'aller de nouveau vers le militarisme allemand ou prussien. Son attitude m'a trop rappelé les jeunes officiers SS, de sinistre mémoire. Si on veut la paix, il faut donner aux jeunes un discours pacifique ${ }^{14}$ !

J'appelle pacifisme paradoxal ce trait caractéristique du Bloc de l'Est qui consiste à militariser la société au nom de la paix. Le pacifisme paradoxal est au cœur de l'idéologie stalinienne du socialisme dans un seul pays qui fut l'idéologie des pays communistes jusqu'à leur effondrement. Le totalitarisme se donne pour mission la construction d'une utopie et d'un homme nouveau en imputant ses propres dysfonctionnements sur un Ennemi extérieur et intérieur. La construction du socialisme dans le seul COMECON est menacée par l'encerclement capitaliste à l'extérieur et les

\footnotetext{
${ }^{13}$ ROMBEAUT, Nestor "Quelques aspects de la vie des travailleurs», in Les Rencontres franco-allemandes, 7, 1960, p. 7.

${ }^{14}$ « Des députés français de retour de RDA nous écrivent », in Rencontres franco-allemandes, 19, 1963, p. 5.
} 
complots de ses agents maléfiques à l'intérieur. Cette mentalité obsidionale justifie d'écrasantes dépenses militaires et la surveillance policière au nom de la paix toujours fragile, toujours menacée. Le militarisme choque les Kéhayan dans la Rue du prolétaire rouge ${ }^{15}$ et Paul Thorez à Artek dans Les enfants modèles ${ }^{16}$. La RDA déploie beaucoup d'effort pour inculquer le militarisme à sa jeunesse qui partirait en première ligne en cas de guerre Est-Ouest. Dans les années 1930 les visiteurs occidentaux les plus critiques mettent fréquemment l'accent à leur retour d'URSS sur la surveillance constante dont ils ont fait l'objet. La Stasi l'a compris et elle est nettement plus discrète. Mais la RDA ne parvient pas à dissimuler les pénuries, la répression et l'embrigadement de sa jeunesse.

\subsection{Les échanges de jeunes}

Les échanges de jeunes ont a priori une vocation plus touristique que les séjours d'économistes ou d'élus qui conservent un caractère professionnel. Le conformisme idéologique de la RDA ressort des témoignages. Les adolescents français effectuent des séjours de travail en entreprise/ repos qui ne semblent pas convaincre comme en témoigne cette lettre de Drancy à Eisenhüttenstadt en 1966 :

il est de notre rôle de faire comprendre aux jeunes gens et aux jeunes filles qu'ils doivent travailler 15 jours dans un esprit différent de l'idée que nous nous faisons du travail en France. En France, nous travaillons toujours pour quelqu'un, il s'agit d'un échange d'argent-travail, qui, dans beaucoup de cas, ressemble à une corvée.

Le sens du travail pour le bien de la collectivité est difficilement compréhensible pour des jeunes gens qui habitent un pays capitaliste aussi, devons-nous préparer ces jeunes en leur faisant sentir que le travail qu'ils accompliront aura un caractère de solidarité d'entre-aide (sic) entre les individus et, qui, librement consenti procure les mêmes joies que les jeux qui, bien souvent ne sont que des passe-temps pour distraire l'homme et ne pas le laisser sombrer dans une oisiveté qui d'après le proverbe est la mère de tous les vices ${ }^{17}$.

\footnotetext{
${ }^{15}$ KÉHAYAN, Nina, KÉHAYAN, Jean, Rue du prolétaire rouge. Deux communistes français en URSS, Paris, Seuil, 1978.

${ }^{16}$ THOREZ, Paul, Les enfants modèles, Paris, Lieu Commun, 1984.

${ }^{17}$ Voyage à Eisenhüttenstadt pour préparer les activités de l'année 1967 et ébaucher celles de 1968 du 7 au 12 décembre p.2. In pochette « RDA : correspondance et semaine de l'amitié (16-24 avril 1966) ». Carton «Eisenhüttenstadt 1961 à 1967».
} 
L'émulation stakhanoviste ne séduit guère les jeunes Français. Le discours de l'élu drancéen reprend la dénonciation de la société de consommation occidentale. Les économies de pénurie des pays de l'Est font de la frugalité vertu.

On apprend ainsi qu'à l'été 1969 sans doute ${ }^{18}$, les enfants de Drancy reçoivent de leurs camarades d'Eisenhüttenstadt une lanterne en carton et papier multicolore, pour prix de leur manque d'ordre et de discipline. L'accompagnatrice prend leur défense, en soulignant que les Français font des efforts pour ranger et nettoyer leur chambre et qu'elle n'apprécie guère ce genre d'humiliation. Le témoignage de Paul Thorez dans Les enfants modèles raconte comment un pionnier de 13 ans est accusé d'houliganisme et condamné à l'autocritique publique pour avoir fait pipi du haut des ruines d'une maison qu'aurait habitée Pouchkine. Les châtiments punissent les fautes idéologiques les plus bénignes. Il décrit les pionniers est-allemands comme les plus disciplinés du Bloc de l'Est. La punition des Drancéens est de toute évidence excessive. Elle dissimule peut-être de l'envie à l'égard de jeunes Français culturellement très libres et pour lesquels ranger une chambre ne présente pas de connotation militariste. Le bismarckisme socialiste qu'exporte la RDA dissimule difficilement la nature totalitaire du régime.

On trouve aussi dans les archives la lettre de Françoise Véron et Françoise Fettu, parties à l'été 1966 dans le cadre des séjours travail-loisir ${ }^{19}$. Leur linge neuf a été dérobé et personne ne semble disposé à les aider. Elles se plaignent d'être laissées à elle-même et affirment avoir déjà été tentées de faire leurs valises. Elles envisagent même d'abréger leur séjour tant elles se sentent déprimées et ajoutent en post-scriptum : « au travail on nous prend pour des chevaux de labour tout en nous faisant de beaux sourire par devant ».

La RDA cherche à rentabiliser les vacances qu'elle offre aux jeunes Français en les faisant travailler. La pression doit être particulièrement forte. Dans Les enfants modèles elle ne paraît pas aussi contraignante. Il est vrai que Paul Thorez décrit le camp d'Artek réservé aux enfants de la nomenklatura qui reçoivent certes des responsabilités et des tâches à accomplir mais où l'essentiel des travaux d'entretien sont effectués par des employés. De surcroît, dans une économie marquée par la sous-productivité, la venue de jeunes occidentaux semble constituer une aubaine.

\footnotetext{
${ }^{18}$ La lettre d'Olga Goulatis, pour autant que je puisse déchiffrer la signature, et écrite de Neu Mukvau, (que je ne suis pas parvenu à localiser), est datée du 20 août sans que l'année ne soit précisée. Etant donné que je l'ai trouvée dans le carton « Eisenhüttenstadt 1968-1970 » il s'agit vraisemblablement de l'été 1969.

${ }^{19}$ Lettre de Françoise Véron à des parents cosignée par Françoise Fettu en date du 11 août 1966. La première est née le 20 juin 1949 à Paris. Elle a donc 17 ans. Elle est inscrite à l'Ecole normale d'institutrice du Bourget et parle allemand. La seconde est née le 15 mai 1945 à Trouville. Elle a 21 ans, et envisage de devenir gardemalade. Ces informations nous sont fournies par leurs fiches respectives. Cfr. carton « Eisenhüttenstadt 1968 à 1970, courrier depuis $1961 »$.
} 


\section{Le tourisme en RDA de la reconnaissance à l'élection de François} Mitterrand (1973-1981)

En 1973 la France reconnaît la RDA, pays que les Français commencent à mieux connaître qu'au cours de la décennie précédente parce que les voyages à l'Est ce sont multipliés et que l'information circule davantage. La question de la dissidence interroge de plus en plus de touristes français qui visitent la Prusse Rouge.

\subsection{La question des libertés et de la dissidence}

En décembre 1972 la RFA et la RDA se reconnaissent mutuellement. Erich Honecker s'efforce de donner une image libérale du régime et annonce devant le Comité central du SED qu'il ne peut y avoir de tabou dans le domaine de l'art et de la littérature si l'on part des positions fermes du socialisme. La Harpe de barbelés ${ }^{20}$ de Wolf Biermann paraît en français en 1972. En 1973 la France reconnait l'Allemagne de l'Est et les Echanges franco-allemands deviennent France-RDA. Ce rapprochement diplomatique coïncide à peu près avec le Programme commun que signent le PC et le PS en 1972. Pour apparaître comme un allié crédible Georges Marchais, conseillé par Jean Kanapa, se pose en défenseur de la démocratie et des libertés. Il s'efforce en même temps, de rester fidèle au Bloc de l'Est qu'il ne peut condamner trop ouvertement au risque de se brouiller avec une fraction de l'électorat et de l'appareil. France-RDA et les comités de jumelage avec la RDA qui restent placés sous la houlette du PCF. Une partie de ses militants souhaiteraient voir l'Allemagne de l'Est concilier socialisme et libertés. C'est de toute évidence la position de JeanPierre Hammer qui a été membre du PCF, des EFA et se présente comme un socialiste humain dans le numéro spécial RDA d'Allemagne d'aujourd'hui de mars-juin 1973 où il soutient Wolf Biermannn en citant Rosa Luxemburg. Or en 1976 Wolf Biermannn est déchu de la nationalité estallemande.

Dans le numéro des Rencontres franco-allemandes de septembre-octobre 1977 Jean-Claude Fouqueau membre du comité de rédaction et du PCF interroge une délégation de syndicalistes français de retour de RDA sur la question des libertés. Un syndicaliste lui répond : «En RDA la première des libertés est celle qui permet à chacun de pouvoir avoir un emploi. Le DROIT au travail est une réalité là-bas ». Fouqueau insiste : «Vous venez de mettre en évidence que ce mot de Liberté n'est pas forcément entendu de la même façon partout, que l'ordre des valeurs semble être différent entre la France et la RDA. Mais la Liberté ne peut se diviser ! ... »

\footnotetext{
${ }^{20}$ BIERMANN, Wolf, Harpe de barbelés, Paris, UGE, 1972.
} 
Le syndicaliste lui rétorque: «Parfaitement et c'est exact que des problèmes existent. Au niveau de la consommation et des biens que l'on peut acheter il reste beaucoup à faire. De gros progrès ont été enregistrés, c'est ainsi que dans la ville où nous séjournions près d'une famille sur deux (43\%) possède une voiture $»^{21}$.

Les syndicalistes de Val-de-Marne qui reviennent de la RDA et adoptent le discours du SED appartiennent certainement à la CGT et au PCF. Cet entretien illustre les clivages internes au Parti communiste et aux EFA. Les $43 \%$ de familles propriétaires de leur voiture illustrent les contradictions d'un régime tiraillé entre un discours anticonsummériste et la volonté d'affronter la concurrence de l'Occident en matière de niveau de vie. En 1973 le PCF fait publier, sous la plume de Bernard Di Crescenzo, Michèle et Jean Tailleur un ouvrage intitulé La RDA un pays hautement développé2 ${ }^{2}$. Les auteurs évoquent la croissance du niveau de vie. Ils affirment par exemple que l'augmentation de la production automobile a atteint 40\% entre 1970 et ... 1975. Un lecteur pressé n'aura peut-être pas eu le temps de voir qu'il s'agit des chiffres du Plan quinquennal. En admettant que le chiffre de $43 \%$ de famille détentrice d'une automobile soit exact on peut en déduire que cette dernière reste un produit relativement peu abordable et que la productivité est faible. Ce chiffre voile la question des défauts techniques des Wartburg 353 et des Trabant 601 qui sont construites pour tenir compte des pénuries. Selon l'historien Alfred Wahl $36,3 \%$ des foyers détiennent une voiture en $1979^{23}$. Le chiffre est vraisemblablement surévalué. Evoquer le droit à l'emploi et l'achat d'automobiles permet d'éluder la vague de répression qui suit la déchéance de nationalité de Wolf Biermann. A l'été 1977 Rudolf Bahro est condamné à huit ans de prison.

Il était certainement préférable pour les Rencontres franco-allemandes d'envoyer des militants convaincus.

\subsection{Le niveau et le mode de vie}

Dans le numéro des Rencontres franco-allemandes comité du Rhône en 1975 une délégation d'animateurs et de directeurs de MJC de retour de Leipzig constate le succès des booms. La ville compte environ 350 orchestres amateurs ainsi qu'un grand nombre de discothèques. La délégation note que les boums ne sont pas interdites et que les éducateurs tentant seulement d'améliorer la qualité de ces soirées, au niveau de l'environnement, du contenu humain et moral. Dans La RDA un pays hautement développé Jean Tailleur qui a écrit la quatrième partie étrangement

\footnotetext{
${ }^{21}$ « Notes de voyage : les entreprises », in Rencontres franco-allemandes, 97, 1977, p. 6.

${ }^{22}$ TAILLEUR, Michèle, TAILLEUR, Jean, DI CRESCENZO, Bernard, La R.D.A. un pays hautement développé, Paris, Editions Sociales, 1973.

${ }^{23}$ WAHL, Alfred, L'Allemagne de 1945 à nos jours, Armand Colin, Paris, 2009, p. 258.
} 
consacrée à « la personnalité socialiste », note qu'à l'occasion de son VIIIème congrès de juin 1971 le SED a annoncé sa volonté de mieux répondre aux aspirations de la jeunesse en matière de danse, de jazz et de Beat. La FDJ, Jeunesse Libres Allemandes, impulse en conséquence un Mouvement des jeunes chanteurs. Jean Tailleur justifie en même temps la lutte idéologique contre les influences occidentales. Il explique que le SED n'a pas renoncé à la formation idéologique ni à l'éducation du goût. La relative libéralisation accordée aux artistes, aux intellectuels et aux modes occidentales s'est doublée d'une forte augmentation des agents de la Stasi passés de 100.000 dans les années 1960 à 180.000 en 1975. Les éducateurs de Leipzig assurent un rôle de surveillance idéologique. L'allusion au contenu humain et moral traduit la pudibonderie qu'observait déjà André Gide dans l'URSS de Staline. Jusqu'en 1968 l'homosexualité était considérée en RDA comme un délit (mineur). En 1975 elle peut encore servir de moyen de chantage.

Erich Honecker n'est pas plus libéral que Walter Ulbricht. En 1965 il avait orchestré la campagne de presse contre «la jeunesse décadente » et les "chevelus », "l'immoralité » et la "décadence américaine ». Il avait même stigmatisé Wolf Biermannn. Devenu Secrétaire général du SED et contraint par la multiplication des contacts entre les populations des deux Allemagnes, à libéraliser le régime, il renforce les moyens de la Stasi. Il reste l'idéologue qu'il a toujours été, pur produit de l'appareil stalinien.

On constate un certain retard du régime est-allemand devant les modes occidentales. Le Beat est né à la fin des années 1950 au Royaume-Uni. A la fin des années 1960 il est sur le déclin. Dans deux ouvrages parus en 1973, La RDA un pays hautement développé et L'enseignement en RDA ${ }^{24}$, le PCF insiste sur la formation de la personnalité socialiste en Allemagne de l'Est. La pénétration des modes occidentales en signe pourtant l'échec.

\subsection{Le modèle économique est-allemand}

Dans La RDA un pays hautement développé Michèle Tailleur présente la RDA comme un pays qui a su concilier augmentation du pouvoir d'achat et stabilité des prix. De toute évidence Erich Honecker accomplit un miracle économique au moment où l'Europe de l'Ouest commence à subir les effets de la stagflation.

En 1977 le syndicaliste interrogé affirme à Jean-Pierre Fouqueau : «des infirmières nous ont dit que "le salaire de début de carrière ne les satisfait pas". Les revendications sont partout posées de façon franche et examinées en fonction de l'intérêt général ${ }^{25}$. En d'autres termes la RDA réussit là où Jacques Chaban-Delmas et Raymond Barre ont échoué. La RDA vise à séduire la

\footnotetext{
${ }^{24}$ BOUVARD, Georges, PELLENQ, Paulette, L'enseignement en RDA, Paris, Editions sociales, 1973. Ce livre est préfacé par Pierre Juquin.

${ }^{25}$ «Notes de voyage : les entreprises», in Rencontres franco-allemandes, 97, 1977, p. 6.
} 
Deuxième gauche, la CFDT, les deloristes et les rocardiens qui prônent déjà l'austérité. Le régime est-allemand cherche à attirer les milieux industriels français. Ces derniers, qui souhaiteraient réduire l'inflation, se heurtent aux revendications salariales et aux syndicats. La RDA cultive son image d'un bismarckisme rouge, à la fois autoritaire et soucieux de l'intérêt ouvrier. Erich Honecker apparaît implicitement sous les traits d'un Frédéric II communiste.

En 1978 Pierre Heinrich, Président du Syndicat National des supermarchés indépendants, de retour de RDA évoque un autre miracle allemand différent de celui de RFA. Il présente l'Allemagne de l'Est comme un pays où les petits commerçants et artisans sont prospères, indépendants, où la propriété est respectée et où les prix sont stables. À lire son témoignage l'Allemagne de l'Est a créé l'ordolibéralisme rouge. Pourtant Erich Honecker a accroit la socialisation des entreprises privées ou semi-privées. Il renforce même la planification: l'économie est placée sous la tutelle du plan à long terme de trente ans, du plan à moyen terme de dix ans, du plan quinquennal et du plan annuel qui ont tous force de loi.

\section{Voyages et tourisme dans un pays ami de la France (1981-1990)}

A partir de 1981, le rapprochement entre Paris et Berlin-Est, resté relativement discret sous la présidence de Valéry Giscard d'Estaing pour des raisons électorales, devient nettement plus visible. En juin 1985 le Premier ministre Laurent Fabius se rend à Berlin-Est et en janvier 1988 Erich Honecker effectue une visite en France. La Prusse rouge s'efforce encore de défendre son image auprès des voyageurs à une époque où le Bloc de l'Est ne séduit plus grand monde.

\subsection{Une économie préservée du chômage}

Alors que la gauche au pouvoir n'a pas pu endiguer la montée du chômage, la RDA est présentée comme une économie créatrice d'emplois notamment grâce à une formation professionnelle efficace.

En 1984 Daniel Magnin membre du bureau national du SNALC note que « l'adéquation entre les besoins de la planification et les désirs des jeunes se fait sans trop de heurts : $80 \%$ d'entre eux exerceront une profession qu'ils ont choisie ou du moins acceptée. Les besoins de main-d'œuvre en RDA étant importants, la tâche des conseillers en est d'autant facilitée ${ }^{26}$. La RDA apparaît encore comme un double de la RFA où la formation professionnelle joue un très grand rôle dans le système éducatif. Le bismarckisme socialiste séduit un cadre du SNALC, peut-être parce qu'il

\footnotetext{
${ }^{26}$ MAGNIN, Daniel, «La formation professionnelle partie intégrante de l'éducation nationale», in Rencontres franco-allemandes, 129, 1984, p. 15.
} 
s'apparente au gaullisme de gauche qu'incarne à cette époque Philippe Séguin, député RPR des Vosges.

\subsection{Le niveau de vie}

On trouve dans les archives d'Argenteuil les notes de Béatrice Cruaud, membre du PS, qui a accompagné le maire communiste Robert Montdargent et son épouse dans un voyage à Dessau ville jumelée, en octobre 1984. Elle constate que l'électro-ménager, le mobilier, l'habillement ressemble à ce qu'ils étaient dans les années 1960 en France. Elle note : «c'est très cher et beaucoup moins sophistiqué que chez nous ; une télé noir et blanc est un luxe au prix comparable à un très beau téléviseur couleur chez nous. Les gens font la queue pour acheter des boules de Noël parce que c'est un objet rare $»^{27}$.

Elle écrit note que le Centre culturel et sportif a été construit par la population. Elle apprend les difficultés de ravitaillement de Dessau, indique que c'est la mairie qui organise l'approvisionnement de la ville en denrées alimentaires et que la question du logement n'est pas encore réglée. Elle remarque les cités de baraquements, la tristesse des paysages des cités est triste, et note que les abords d'immeubles peu ou pas aménagés. Elle ajoute : " nous avons demandé à visiter l'appartement de l'adjoint au maire : les chambres sont très petites. Nous avons eu très peu de contacts avec la population ${ }^{28}$.

Son témoignage présente l'intérêt d'être détaillé. Au début des années 1980 la pénurie alimentaire s'est aggravée. L'URSS réduit ses livraisons d'hydrocarbure à la RDA en 1981 et entraîne cette dernière dans une course aux armements dont elle n'a pas les moyens. Faute de devises le régime est-allemand n'a plus les moyens d'importer de l'Ouest de l'alimentation pour le bétail et l'élevage s'effondre.

L'habitat est un échec malgré le volontarisme du régime en la matière. Entre 1976 et 1981, 750.000 logements ont été construits ou rénovés contre 600000 entre 1971 et $1975^{29}$.

Elle interprète la réalisation du Centre culturel et sportif comme une forme d'exploitation bureaucratique de la population et non sous les traits de l'émulation socialiste. Le samedi socialiste ne semble guère l'enchanter. Elle n'a guère ne doute sur l'état d'esprit de la population et suggère de manière très diplomatique qu'elle n'adhère pas au régime.

\footnotetext{
${ }^{27}$ Archives municipales d'Argenteuil, dossier 46W28, Béatrice Cruaud, « Séjour à Dessau 26-27-28 octobre $1984 »$.

${ }^{28}$ Ibidem.

${ }^{29}$ WAHL, Alfred, L'Allemagne de 1945 à nos jours, Paris, Armand Colin, 2009, p. 259.
} 


\subsection{Voyages et libertés}

En septembre 1984 une délégation de juristes se rend en RDA pour y discuter de la situation des droits de l'homme et des libertés ${ }^{30}$. Le compte-rendu est présenté par Laure Perrin magistrate membre de la présidence de France-RDA. Elle écrit qu'une campagne est organisée en France sur la question des libertés en Allemagne de l'Est. L'association est régulièrement saisie de courriers d'Amnesty International sollicitant la défense de prisonniers politiques. Laure Perrin fait notamment allusion à l'ouvrage paru en 1983 RDA : Atteintes à la liberté d'expression en République démocratique allemande ${ }^{31}$.

La délégation a engagé une discussions avec ses partenaires, dont l'identité est reste floue. Il s'agit vraisemblablement de RDA-France puisqu'elle cite le Docteur Scholz qui la préside et fut le premier ambassadeur d'Allemagne de l'Est en France. Ce dernier a expliqué qu'en RDA les droits fondamentaux des travailleurs à vivre dans la paix, le droit au travail, à l'espace d'habitation, la participation des citoyens à la vie publique étaient respectés. Les Français ont également rencontré des représentants du Front national, coalition à liste unique destinée à créer l'illusion du multipartisme. Ils ont discuté de la petite délinquance et des soins aux personnes âgées. La question des libertés publiques gêne toujours la RDA.

\section{Conclusion}

Le tourisme en RDA est surveillé et très organisé. Le voyage dans l'Allemagne de l'Est ne saurait être comparé à un séjour dans la libre RFA. Dans un pays très fermé comme la RDA le régime cherche à séduire les visiteurs et à donner l'image d'un bismarckisme socialiste. Une Prusse Rouge qui aurait opéré un second miracle économique allemand au service de la classe populaire et de l'intérêt général. Aussi contradictoire qu'elle puisse paraitre l'image d'un bismarckisme socialiste vise à séduire les investisseurs français sans rompre avec son identité communiste. Le bismarckisme socialiste cherche à éviter les scandales qu'ont produit les témoignages comme le Retour de l'URSS d'André Gide. Le bismarckisme rouge, à la fois bourgeois et socialiste, autoritaire et démocratique, pacifiste et militariste, ne semble avoir convaincu que les convaincus.

\footnotetext{
${ }^{30}$ PERRIN, Laure, « Des juristes français en RDA », in Rencontres franco-allemandes, 129, 1984, p. 6.

${ }^{31}$ AMNESTY INTERNATIONAL - RDA, Atteintes à la liberté d'expression en République démocratique allemande, Paris, Éd. francophones d'Amnesty international, 1983.
} 


\section{L'AUTEUR}

William RICHIER, professeur d'histoire-géographie dans l'enseignement secondaire, prépare une thèse sur les jumelages entre la France et la RDA entre 1959 et 1990. Il est également correspondant de l'Institut d'histoire du temps présent. Il a participé aux enquêtes sur « la guerre froide vue d'en bas », « la sociologie des élites culturelles locales » et collabore actuellement à la recherche " accueillir les étrangers en France ». Il est versé dans l'histoire des idées politiques.

URL: < http://www.studistorici.com/progett/autori/\#Richier > 